\title{
ADAPTIVE CONTROL STRATEGIES AND DEPENDENCE OF FINITE TIME RUIN ON THE PREMIUM LOADING
}

\author{
Vsevolod K. Malinovskii
}

\begin{abstract}
The paper is devoted to risk theory insight into the problem of asset-liability and solvency adaptive management. Two adaptive control strategies in the multiperiodic insurance risk model composed of chained classical risk models are introduced and their performance in terms of probability of ruin is examined. The analysis is based on an explicit expression of the probability of ruin within finite time in terms of Bessel functions. Dependence of that probability on the premium loading, either positive or negative, is basic technical result of independent interest.
\end{abstract}

\section{Introduction}

Insurance industry is subject to intensive regulation. Supervision authorities watch compliance with the regulatory principles designed to balance the solvency and equity requirements. According to the regulatory principles, each insurer must report his financial position yearly or even more often, if required.

The insurance process is viewed therefore as a series of successive insurance years. Each year starts with manager's control intervention which fine-tunes tariffs, reserves and other operational characteristics of the probability mechanism of insurance. Its influence remains in force throughout the whole insurance year, i.e., until the next report and subsequent control intervention.

The insurance regulation and supervision will be blind without a comprehensive model, or a set of models, describing the probability mechanism of insurance within an operating period. Suitable is the Lundberg's collective risk model which considers the net result of the risk business of an insurer from the position of a "remote" observer, and is often named the main achievement of the 20-th century risk theory.

The background of the present paper is a general multiperiodic controlled risk model introduced in [13] and composed of chained singleperiodic risk models. The trajectory of a general multiperiodic insurance process with annual accounting and

Key words and phrases. Multiperiodic insurance risk model, equity-solvency balance, adaptive control, classical risk model, finite time ruin probabilities, Bessel functions, premium loading. 
subsequent annual control may be diagramed as

$$
w_{0} \underbrace{\stackrel{\gamma_{0}}{\longrightarrow} u_{0} \stackrel{\pi_{1}}{\longrightarrow} w_{1}}_{\text {1-st year }} \cdots \stackrel{\pi_{k-1}}{\longrightarrow} w_{k-1} \underbrace{\stackrel{\gamma_{k-1}}{\longrightarrow} u_{k-1} \stackrel{\pi_{k}}{\longrightarrow} w_{k}}_{k \text {-th year }} \cdots .
$$

According to this diagram (for $k=1,2, \ldots$ ), at the end of the $(k-1)$-th year the state variable $w_{k-1}$ is observed; it describes the insurer's position at that time. Then, at the beginning of the $k$-th year the control rule $\gamma_{k-1}$ is applied to choose the control variable $u_{k-1}$. Thereupon the $k$-th year probability mechanism of insurance unfolds; the transition function of this mechanism is denoted by $\pi_{k}$. It defines the insurer's position at the end of the $k$-th year.

In that framework, consider the adaptive control strategy introduced in [14]. It is defined as follows. At the time of selection of the incoming year control, let $t$ be the next-year length and $z$ be a deviation, either positive or negative, of the past-year-end risk reserve from a certain value $u_{\lambda, \mu}(\alpha, t)$ called target capital value. Case $z<0$ means deficit, case $z>0$ means surplus. Let the annual control be twofold, i.e., it consists of assigning of the starting capital and premium loading according to the rule

$$
u_{z, t}=u_{\lambda, \mu}(\alpha, t)+z \quad \text { and } \quad \tau_{z, t}=-\frac{\mu}{\lambda t} z .
$$

That choice of $u_{z, t}$ means starting with the initial capital equal to the past-year-end risk reserve, without regard to either $z$ is a surplus over $u_{\lambda, \mu}(\alpha, t)$, or a deficit. The latter is appreciated in the choice of the adaptive loading $\tau_{z, t}$, positive in the deficit case $(z<0)$ and negative in the surplus case $(z>0)$.

The target capital value corresponding to a level $\alpha \in(0,1)$ is such a value $u=u_{\lambda, \mu}(\alpha, t)$ which satisfies the "neutral-loading" equation

$$
\boldsymbol{\psi}_{t}(u ; 0)=\alpha,
$$

where $\boldsymbol{\psi}_{t}(u ; \tau)$ is the probability of ruin within time $t$, when premium loading is $\tau$.

Since under the control (1) the risk reserve (see (5) and (6) below) is

$$
R_{s}\left(u_{z, t}, \tau_{z, t}\right)=\left(u_{\lambda, \mu}(\alpha, t)+z\right)+\left(1-\frac{\mu}{\lambda t} z\right) \frac{\lambda}{\mu} s-\sum_{i=1}^{N(s)} Y_{i}, \quad 0 \leqslant s \leqslant t,
$$

one has

$$
\mathrm{E} R_{t}\left(u_{z, t}, \tau_{z, t}\right)=u_{\lambda, \mu}(\alpha, t) \text { for any } z,
$$

and the control (1) induces the capital at the time $t$ (i.e., at the year-end of a single period) to be equal "in the average" to the target value $u_{\lambda, \mu}(\alpha, t)$. That observation justifies the name of target capital value.

Bearing in mind equity considerations, the negative premium loading in case of surplus $(z>0)$ does not seem unrealistic, particularly to practical people. For instance, Benjamin in his discussion of [6] noted: "One would almost think that this is against the interests of the policyholders, because it pays out money away from the company. But most life insurance companies pay a dividend which is based on their surplus and they pay a bonus to their policyholders calculated on the same basis so that the two are brought into line."

Voluntary or directive decrease of premium loading may be interpreted as, e.g., redistribution of excessive premiums of past years, or stimulation of inflow of clients. Involuntary (e.g., due to erroneous assessment of incompletely known future risk) decrease tightly connected with insurance cycles will be analyzed elsewhere. 
The solvency performance of the $n$-year strategy is commonly measured by the probability of ruin

where $^{1}$

$$
\mathrm{P}\{\text { ruin within } n \text { years }\}=\sum_{k=1}^{n} \mathrm{P}\{\text { first ruin in year } k\},
$$

$$
\begin{aligned}
& \mathrm{P}\{\text { first ruin in year } k\}=\int \pi_{t}\left(d x_{1}, \text { no ruin } \mid u_{z(u), t}, \tau_{z(u), t}\right) \\
& \ldots \int \pi_{t}\left(d x_{k-1} \text {, no ruin } \mid u_{z\left(x_{k-2}\right), t}, \tau_{z\left(x_{k-2}\right), t}\right) \int \psi_{t}\left(u_{z\left(x_{k-1}\right), t}, \tau_{z\left(x_{k-1}\right), t}\right), \\
& z(x)=x-u_{\lambda, \mu}(\alpha, t) \text { and } \\
& \pi_{t}(A, \text { no ruin } \mid u, \tau)=\mathrm{P}\left\{R_{t}(u, \tau) \in A, \underset{0<s \leqslant t}{\inf } R_{s}(u, \tau)>0\right\} .
\end{aligned}
$$

The ultimate goal of the paper is to analyze these expressions.

The structure of the paper is as follows. In Section 2 we formulate basic results on the year-end risk reserve distribution and ruin probability. Bearing in mind the adaptive control strategy (1) and its alternative with borrowing, in Section 3 we scrutinize the monotone character of dependence of the probability of ruin on the premium loading. In Section 4 we obtain upper bounds on the probabilities of ruin for theses strategies. Section 5 contains auxiliary results.

\section{Risk reserve and ruin in singleperiodic risk model}

The classical (Poisson-Exponential) risk model which we select here to be singleperiodic risk model, comes from the random variables $\left\{T_{i}\right\}_{i \geqslant 1}$ and $\left\{Y_{i}\right\}_{i \geqslant 1}$, i.i.d. and mutually independent, where $T_{i}$ are the interclaim times and $Y_{i}$ are the amounts of claims, exponentially distributed with parameters $\lambda>0$ and $\mu>0$, respectively. These random variables generate the risk reserve process

$$
R_{t}(u, \tau)=u+c t-V(t),
$$

where $V(t)=\sum_{i=1}^{N(t)} Y_{i}, t \geqslant 0, u>0$ is the initial risk reserve, $c>0$ is the risk premium rate and $N(t)$ is the largest $n$ for which $\sum_{i=1}^{n} T_{i} \leqslant t$ (we put $N(t)=0$ if $\left.T_{1}>t\right)$. For $t \geqslant 0$, it generates Poisson process of density $\lambda$.

If the premium rate $c$ is assigned according to the expected value principle, the amount

$$
\tau=\left(c \mathrm{E} T_{1}-\mathrm{E} Y_{1}\right) / \mathrm{E} Y_{1}=c \mu / \lambda-1
$$

is called the relative loading (when $\tau>0$, it is called the relative safety loading), and

$$
c=(1+\tau) \lambda / \mu \text {. }
$$

The sign of the loading $\tau$ is an important discriminating factor in the traditional ruin problem. The condition $\tau>0$, or, equivalently, $c \mathrm{E} T_{1}>\mathrm{E} Y_{1}$, implies that the insurance business tends to be profitable in the long run. Indeed, $c T_{i}$ is the premium acquired during the $i$-th "step", where "step" is the time between $(i-1)$-th and $i$-th claims, and $Y_{i}$ is the corresponding payout. Since the "steps" are independent and probabilistically identical, the above inequality for expectations means persistency of the "profitable steps", when claim payout is less than premium acquired.

\footnotetext{
${ }^{1}$ We assume here that $t, \lambda$ and $\mu$ are identical throughout insurance years only for notational simplicity.
} 
Ruin occurs when $R_{s}(u, \tau)<0$, and the probabilities of ultimate ruin and of ruin within time interval $t$ are respectively

$$
\psi(u, \tau)=\mathrm{P}\left\{\inf _{0<s<\infty} R_{s}(u, \tau)<0\right\}, \quad \psi_{t}(u, \tau)=\mathrm{P}\left\{\inf _{0<s \leqslant t} R_{s}(u, \tau)<0\right\} .
$$

The results formulated further in this section, some of them known, make the exposition self-contained, contribute to reader's convenience and are prelusive for the fundamental results of Section 3 .

2.1. Year-end risk reserve distribution. Essential is the following theorem.

THEOREM 2.1. In the classical risk model, for $x \in \mathrm{R}$,

$$
\mathrm{P}\left\{R_{t}(u, \tau) \leqslant x\right\}= \begin{cases}1, & x>u+c t \\ 1-e^{-\lambda t}-e^{-\lambda t}(\lambda \mu t)^{1 / 2} & \\ \times \int_{0}^{u+c t-x} z^{-1 / 2} I_{1}(2 \sqrt{\lambda \mu t z}) e^{-\mu z} d z, & x \leqslant u+c t\end{cases}
$$

where $I_{1}(\cdot)$ is the modified Bessel function of unit order.

LEMMA 2.1 (Seal [18]). In the classical risk model, for $x \geqslant 0$,

$$
\begin{aligned}
\mathrm{P}\{V(t) \leqslant x\} & =e^{-\lambda t}+e^{-\lambda t} \sum_{n=1}^{\infty} \frac{(\lambda t)^{n}}{n !} \frac{\mu^{n}}{\Gamma(n)} \int_{0}^{x} e^{-\mu z} z^{n-1} d z \\
& =e^{-\lambda t}+e^{-\lambda t}(\lambda \mu t)^{1 / 2} \int_{0}^{x} z^{-1 / 2} I_{1}(2 \sqrt{\lambda \mu t z}) e^{-\mu z} d z,
\end{aligned}
$$

where $I_{1}(\cdot)$ is the modified Bessel function of unit order.

Proof of Lemma 2.1. Apply the total probability formula with respect to the decomposition of $\Omega$ by non-intersecting measurable sets $\{N(t)=n\}, n=$ $0,1,2, \ldots$ Bearing in mind that $\mathrm{P}\{N(t)=n\}=e^{-\lambda t}(\lambda t)^{n} / n$ !, for $F(x, t)=$ $\mathrm{P}\{V(t) \leqslant x\}$ one has $F(x, t)=e^{-\lambda t}+\sum_{n=1}^{\infty} e^{-\lambda t} \frac{(\lambda t)^{n}}{n !} P_{Y}^{* n}(x)$. The sum $\sum_{i=1}^{n} Y_{i}$ of i.i.d. exponential summands is Gamma-distributed (see, e.g., [8], Chapter I, Section 3), and

$$
P_{Y}^{* n}(x)=\frac{\mu^{n}}{\Gamma(n)} \int_{0}^{x} e^{-\mu z} z^{n-1} d z, \quad n=1,2, \ldots
$$

Bearing in mind discontinuity in zero, introduce $f(x, t)=\frac{\partial}{\partial x} F(x, t)$ for $x>0$ and note (see (53)) that

$$
\begin{aligned}
f(x, t) & =\lambda \mu t e^{-\lambda t-\mu x} \sum_{n=1}^{\infty} \frac{(\lambda \mu t x)^{n-1}}{n !(n-1) !} \\
& =(\lambda \mu t)^{1 / 2} x^{-1 / 2} e^{-\lambda t-\mu x} I_{1}(2 \sqrt{\lambda \mu t x}), \quad x>0 .
\end{aligned}
$$

To get the second expression (7), put it in $F(x, t)=e^{-\lambda t}+\int_{0}^{x} f(z, t) d z$.

Proof of TheOrem 2.1. It follows directly from the equality

$$
\mathrm{P}\left\{R_{t}(u, \tau) \leqslant x\right\}= \begin{cases}1-\mathrm{P}\{V(t)<u+c t-x\}, & x \leqslant u+c t \\ 1, & x>u+c t\end{cases}
$$

and Lemma 2.1. 
THEOREM 2.2. In the classical risk model

$$
\begin{aligned}
\mathrm{E}(V(t)) & =\frac{\lambda t}{\mu}, & \mathrm{E}\left(V(t)^{2}\right) & =\frac{\lambda t(\lambda t+2)}{\mu^{2}}, \\
\mathrm{D}(V(t)) & =2 \frac{\lambda t}{\mu^{2}}, & \mathrm{E}\left(V(t)^{3}\right) & =\frac{\lambda t\left((\lambda t)^{2}+6(\lambda t+1)\right)}{\mu^{3}} .
\end{aligned}
$$

Proof. Bearing in mind Theorem 2.1 and equations

$$
\begin{aligned}
& \int_{0}^{\infty} z^{1 / 2} I_{1}\left(a z^{1 / 2}\right) e^{-b z} d z=\frac{a}{2 b^{2}} \exp \left\{\frac{a^{2}}{4 b}\right\} \\
& \int_{0}^{\infty} z^{3 / 2} I_{1}\left(a z^{1 / 2}\right) e^{-b z} d z=\frac{a}{8 b^{4}}\left(a^{2}+8 b\right) \exp \left\{\frac{a^{2}}{4 b}\right\}, \\
& \int_{0}^{\infty} z^{5 / 2} I_{1}\left(a z^{1 / 2}\right) e^{-b z} d z=\frac{a}{32 b^{6}}\left(a^{4}+24 a^{2} b+96 b^{2}\right) \exp \left\{\frac{a^{2}}{4 b}\right\}
\end{aligned}
$$

(see, e.g., [10], equation 6.643.2) with $a=2 \sqrt{\lambda \mu t}, b=\mu$, the proof is straightforward. For example,

$$
E(V(t))=\int_{0}^{\infty} x f(x, t) d x=e^{-\lambda t}(\lambda \mu t)^{1 / 2} \int_{0}^{\infty} z^{1 / 2} I_{1}(2 \sqrt{\lambda \mu t z}) e^{-\mu z} d z=\frac{\lambda t}{\mu} .
$$

2.2. Annual ruin probability. Appearingly different methods lead to the same explicit expression for the probability of ruin $\boldsymbol{\psi}_{t}(u, \tau)$ in the classical risk model (see, e.g., [16], Chapter 5, Section 5.5, [20], Chapter 7, Section 35).

To have the formula (10) below, Asmussen (see formulas (7.1)-(7.4) in [3] and Proposition 1.3 in [4], where more particulars of the proof are given ${ }^{2}$ ) followed Seal (see formula (21) in [18]) and applied queueing interpretation of ruin first made explicit by Prabhu [16]. Drekic and Willmot (see Section 2 in [7]) started from the so-called Gerber-Shiu defective renewal equation in case of Poisson claims arrival and general claims amounts.

The same result may be obtained from quite different premises. Dealing with more general claims arrival than merely Poisson, and with exponential claim amounts $^{3}$, Malinovskii (see Section 2 in [11]) embarked from the Laplace transform of $\boldsymbol{\psi}_{t}(u, \tau)$ (see Theorem 2.3 below). In this section we revisit the approach of $[\mathbf{1 1}]$, starting from the following basic result.

TheOREM 2.3 (Theorem 1 in [11] and Theorem 2.4 in [12]). Let the claim sizes $\left\{Y_{i}\right\}_{i \geqslant 1}$ and the interclaim times $\left\{T_{i}\right\}_{i \geqslant 1}$ be i.i.d. and mutually independent. Let $Y_{1}$ be exponential with a parameter $\mu>0$ and the Laplace transform of $T_{1}$ be $\gamma_{T}(\alpha)=\int_{0}^{\infty} e^{-\alpha z} P_{T}(d z)$. Then

$$
\alpha \int_{0}^{\infty} e^{-\alpha t} \boldsymbol{\psi}_{t}(u, \tau) d t=y(\alpha) \exp \{-u \mu(1-y(\alpha))\}, \quad \alpha>0,
$$

where $y(\alpha)$ is a solution of the equation

$$
y(\alpha)=\gamma_{T}(\alpha+c \mu(1-y(\alpha))), \quad \alpha>0 .
$$

\footnotetext{
${ }^{2}$ Several misprints contained in $[\mathbf{3}]$ and pointed in $[\mathbf{5}]$ are eliminated in $[\mathbf{4}]$ as well.

${ }^{3}$ By means of a straightforward development of the technique introduced in [11], this result was generalized on certain finite mixtures of exponential claims in [?].
} 
Corollary 2.1 (Remark 2 on p. 127 in [11] and Theorem 2.3 in [12]). In the classical risk model

$$
\boldsymbol{\psi}_{t}(u, \tau)=\boldsymbol{\psi}(u, \tau)-\frac{1}{\pi} \int_{0}^{\pi} f(x, u, t) d x
$$

for any $u>0$, where

$$
\psi(u, \tau)= \begin{cases}(\lambda / c \mu) \exp \{-u \mu(1-\lambda / c \mu)\}, & c \mu / \lambda>1 \\ 1, & c \mu / \lambda \leqslant 1\end{cases}
$$

and

$$
\begin{aligned}
f(x, u, t) & =(\lambda / c \mu)(1+\lambda / c \mu-2 \sqrt{\lambda / c \mu} \cos x)^{-1} \\
& \times \exp \{u \mu(\sqrt{\lambda / c \mu} \cos x-1)-\lambda t(c \mu / \lambda)(1+\lambda / c \mu-2 \sqrt{\lambda / c \mu} \cos x)\} \\
& \times[\cos (u \mu \sqrt{\lambda / c \mu} \sin x)-\cos (u \mu \sqrt{\lambda / c \mu} \sin x+2 x)] .
\end{aligned}
$$

REMARK 2.1. It is noteworthy that the expression in the right hand side of (10) depends on $c, \mu$ and $\lambda$ through $u \mu, \lambda t$ and the ratio $\lambda / c \mu$ only.

Proof OF COROLlary 2.1. For $T_{1}$ exponential with parameter $\lambda>0$, one has $\gamma_{T}(\alpha)=\lambda /(\lambda+\alpha)$, and the solution of the equation (9) is straightforward:

$$
y(\alpha)=\sqrt{\lambda / c \mu}(a-b)(\sqrt{a+\alpha}+\sqrt{b+\alpha})^{-2},
$$

where $a=(\sqrt{c \mu}+\sqrt{\lambda})^{2}, b=(\sqrt{c \mu}-\sqrt{\lambda})^{2}$.

Expanding exponent in the equation (8), obtain

$$
\begin{aligned}
\alpha \int_{0}^{\infty} e^{-\alpha t} \boldsymbol{\psi}_{t}(u, \tau) d t=y(\alpha) \exp \{-u \mu(1-y(\alpha))\} \\
\quad=e^{-u \mu} \sum_{n \geqslant 0} \frac{(u \mu)^{n}}{n !} y(\alpha)^{n+1} \\
\quad=e^{-u \mu} \sum_{n \geqslant 0} \frac{(u \mu)^{n}}{n !} \frac{(a-b)^{n+1}}{(\sqrt{a+\alpha}+\sqrt{b+\alpha})^{2(n+1)}}\left(\frac{\lambda}{c \mu}\right)^{(n+1) / 2}, \quad \alpha>0
\end{aligned}
$$

Recollect (see equation (58) in Lemma 5.2) that

$$
\left((a-b)(\sqrt{a+\alpha}+\sqrt{b+\alpha})^{-2}\right)^{n}
$$

is the Laplace transform of the function

$$
n e^{-(a+b) x / 2} x^{-1} I_{n}((a-b) x / 2),
$$

and the right hand side of (12) is the Laplace transform of the function

$$
f(x)=e^{-u \mu} \sum_{n \geqslant 0} \frac{(u \mu)^{n}}{n !}\left(\frac{\lambda}{c \mu}\right)^{(n+1) / 2} \frac{n+1}{x} e^{-(c \mu+\lambda) x} I_{n+1}(2 x \sqrt{c \lambda \mu}) .
$$
yields

Regarding the left hand side of (12), note that $\boldsymbol{\psi}_{t}(u, \tau)=\int_{0}^{t} f(x) d x$, which

$$
\begin{aligned}
\boldsymbol{\psi}_{t}(u, \tau)=e^{-u \mu} \sum_{n \geqslant 0} \frac{(u \mu)^{n}}{n !}\left(\frac{\lambda}{c \mu}\right)^{(n+1) / 2} & \\
& \times \int_{0}^{\lambda t} \frac{n+1}{x} e^{-(1+c \mu / \lambda) x} I_{n+1}(2 x \sqrt{c \mu / \lambda}) d x .
\end{aligned}
$$


The explicit expression for $\boldsymbol{\psi}_{t}(u, \tau)$ is derived; further concern is to represent it in the equivalent form (10), which is generally believed (see, e.g., $[\mathbf{3}],[\mathbf{4}]$ ) to be better suited for numerical calculations ${ }^{4}$.

Apply Lemma 5.1 which yields

$$
\boldsymbol{\psi}_{t}(u, \tau)= \begin{cases}e^{-u \mu} \frac{\lambda}{c \mu} \sum_{n \geqslant 0}\left(\frac{\lambda}{c \mu}\right)^{n} \frac{(u \mu)^{n}}{n !}\left(1-v_{n}(c \mu t)\right), & c \mu / \lambda>1, \\ e^{-u \mu} \frac{\lambda}{c \mu} \sum_{n \geqslant 0}\left(\frac{\lambda}{c \mu}\right)^{n} \frac{(u \mu)^{n}}{n !}\left(\left(\frac{c \mu}{\lambda}\right)^{n+1}-v_{n}(c \mu t)\right), & c \mu / \lambda \leqslant 1,\end{cases}
$$

where

$$
v_{n}(c \mu t)=\frac{2}{\pi}\left(\frac{c \mu}{\lambda}\right)^{n / 2} \int_{0}^{\pi} \sin x \sin (n+1) x e^{-c \mu A(x) t} A(x)^{-1} d x
$$

and $A(x)=1+\lambda / c \mu-2 \sqrt{\lambda / c \mu} \cos x$.

Regarding (14), note first that

$$
e^{-u \mu} \frac{\lambda}{c \mu} \sum_{n \geqslant 0}\left(\frac{\lambda}{c \mu}\right)^{n} \frac{(u \mu)^{n}}{n !}=(\lambda / c \mu) \exp \{-u \mu(1-\lambda / c \mu)\}
$$

in case $c \mu / \lambda>1$, and

$$
e^{-u \mu} \frac{\lambda}{c \mu} \sum_{n \geqslant 0}\left(\frac{\lambda}{c \mu}\right)^{n} \frac{(u \mu)^{n}}{n !}\left(\frac{c \mu}{\lambda}\right)^{n+1}=1
$$

in case $c \mu / \lambda \leqslant 1$. Then put (15) in

$$
e^{-u \mu} \frac{\lambda}{c \mu} \sum_{n \geqslant 0}\left(\frac{\lambda}{c \mu}\right)^{n} \frac{(u \mu)^{n}}{n !} v_{n}(c \mu t)
$$

and, bearing in mind that $\sin (n+1) x=\left(e^{i(n+1) x}-e^{-i(n+1) x}\right) / 2 i$, obtain for $a=$ $u \mu \sqrt{\lambda / c \mu}$

$$
\begin{aligned}
& \sum_{n \geqslant 0} \frac{a^{n}}{n !} \sin (n+1) x=\frac{1}{2 i} \sum_{n \geqslant 0} \frac{a^{n}}{n !}\left(e^{i(n+1) x}-e^{-i(n+1) x}\right) \\
&=\frac{1}{2 i}\left(e^{i x} e^{a e^{i x}}-e^{-i x} e^{a e^{-i x}}\right)=\frac{1}{2 i}\left(e^{a \cos x+i(x+a \sin x)}-e^{a \cos x-i(x+a \sin x)}\right) \\
&=e^{a \cos x} \sin (x+a \sin x) .
\end{aligned}
$$

It eliminates summation. To complete the proof, note that $2 \sin x \sin (x+a \sin x)=$ $\cos (a \sin x)-\cos (a \sin x+2 x)$.

REMARK 2.2. The equation (13) is essentially the same as (2.7) in [7] and (7.2) in [3]. It is not only an important intermediate step in the proof of Corollary 2.1, but also an essential starting point in further exposition.

2.3. Year-end risk reserve and ruin. For $x \in \mathrm{R}$ and Borel set $A$, consider

$$
\begin{aligned}
\pi_{t}(A, \operatorname{ruin} \mid u, \tau) & =\mathrm{P}\left\{R_{t}(u, \tau) \in A, \inf _{0<s \leqslant t} R_{s}(u, \tau)<0\right\}, \\
\pi_{t}(A, \text { no ruin } \mid u, \tau) & =\mathrm{P}\left\{R_{t}(u, \tau) \in A, \inf _{0<s \leqslant t} R_{s}(u, \tau)>0\right\} \\
& =\mathrm{P}\left\{R_{t}(u, \tau) \in A\right\}-\pi_{t}(A, \operatorname{ruin} \mid u, \tau)
\end{aligned}
$$

\footnotetext{
${ }^{4}$ The analysis advocating convenience of (13) for numerical calculations see in [15].
} 
and note that

$$
0 \leqslant \pi_{t}(A, \text { ruin } \mid u, \tau) \leqslant \boldsymbol{\psi}_{t}(u ; \tau),
$$

and

$$
0 \leqslant \pi_{t}(A, \text { no ruin } \mid u, \tau) \leqslant \mathrm{P}\left\{R_{t}(u, \tau) \in A\right\} .
$$

More delicate analysis is based on the joined distribution of the time of ruin $\omega$ and the deficit at ruin $\gamma$, explicit in the classical model (see, e.g., [9]) since

$$
\pi_{t}(A, \text { ruin } \mid u, \tau)=\int_{0}^{\infty} \int_{0}^{t} \mathrm{P}\{\omega \in d s, \gamma \in d y\} \mathrm{P}\left\{R_{t-s}(-y, \tau) \in A\right\} .
$$

We will not go into further details here ${ }^{5}$.

\section{Bessel functions, ruin and a random walk process}

For brevity sake, in Section 3 apply the following re-parametrization: put

$$
\varphi_{t}(u, p)=e^{-u \mu} \sum_{n \geqslant 0} \frac{(u \mu)^{n}}{n !}\left(\frac{p}{1-p}\right)^{\frac{n+1}{2}} \int_{0}^{\lambda t} \frac{n+1}{x} e^{-x / p} I_{n+1}(2 x \sqrt{(1-p) / p}) d x
$$

and $\varphi(u, p)=\varphi_{\infty}(u, p)$ for $0 \leqslant p \leqslant 1$ and note that

$$
\boldsymbol{\psi}_{t}(u, \tau)=\varphi_{t}(u, p(\tau)) \text { and } \quad \boldsymbol{\psi}(u, \tau)=\varphi(u, p(\tau))
$$

where

$$
p(\tau)=\frac{1}{2+\tau} .
$$

Equation (13) implies (17) immediately since $\tau=c \mu / \lambda-1 \in(-1, \infty)$ (see equation $(6)), p(\tau)=1 /(2+\tau)=\lambda /(c \mu+\lambda), c \mu / \lambda=(1-p(\tau)) / p(\tau)$ and $1+c \mu / \lambda=1 / p(\tau)$.

It is noteworthy that $0<p(\tau)<1$, and the inequalities $p(\tau)<1 / 2$ and $p(\tau) \geqslant 1 / 2$ are equivalent to $\tau>0$ and $\tau \leqslant 0$, respectively.

3.1. Bessel functions and ruin probabilities. Rewrite $\varphi_{t}(u, p)$ as

$$
\varphi_{t}(u, p)=e^{-u \mu} \sum_{n \geqslant 0} \frac{(u \mu)^{n}}{n !} \int_{0}^{\lambda t} v_{n+1}(x, p) d x
$$

where

$$
v_{n+1}(x, p)=\left(\frac{p}{1-p}\right)^{\frac{n+1}{2}} \frac{n+1}{x} e^{-x / p} I_{n+1}(2 x \sqrt{(1-p) / p}) .
$$

Apply equality (58) and note that $\int_{0}^{\infty} v_{n+1}(x, p) d x=\left(\frac{p}{1-p}\right)^{n+1} \mathbf{1}_{\{p<1 / 2\}}+$ $\mathbf{1}_{\{p \geqslant 1 / 2\}}$. Furthermore,

$$
\begin{aligned}
\varphi(u, p)=e^{-u \mu} \sum_{n \geqslant 0} \frac{(u \mu)^{n}}{n !} \int_{0}^{\infty} v_{n+1}(x, p) d x & \\
=e^{-u \mu} \sum_{n \geqslant 0} \frac{(u \mu)^{n}}{n !} & \left(\frac{p}{1-p}\right)^{n+1} \mathbf{1}_{\{p<1 / 2\}}+e^{-u \mu} \sum_{n \geqslant 0} \frac{(u \mu)^{n}}{n !} \mathbf{1}_{\{p \geqslant 1 / 2\}} \\
& =\frac{p}{1-p} \exp \left\{-u \mu \frac{1-2 p}{1-p}\right\} \mathbf{1}_{\{p<1 / 2\}}+\mathbf{1}_{\{p \geqslant 1 / 2\}} .
\end{aligned}
$$

\footnotetext{
${ }^{5}$ In $[3]$ it is shown that in the Poisson-Exponential model $\omega$ is independent on $\gamma$; the latter is exponential with parameter $\mu$.
} 
It is noteworthy that the right hand side of (21) with $p=p(\tau), \tau=c \mu / \lambda-1$, coincides with (11), which is the well known Cramér formula for the ultimate ruin probability. Indeed, for $p(\tau) \geqslant 1 / 2$, which equals to $\tau \leqslant 0, \varphi(u, p(\tau))$ is unit; in case $p(\tau)<1 / 2$ of positive loading, i.e., $\tau>0$, the factor $\mu(1-\lambda / c \mu)=$ $\mu(1-2 p(\tau)) /(1-p(\tau))$ is usually referred to as the Lundberg exponent, or the adjustment coefficient, $\varkappa=\mu \tau /(1+\tau)>0$. The factor $\lambda / c \mu=p(\tau) /(1-p(\tau))$ is referred to as the Cramér-Lundberg constant $C=1 /(1+\tau)>0$.

Introduce

$$
f_{n+1}(x, p)=\left(\frac{1-p}{p}\right)^{n+1} v_{n+1}(x, p) \mathbf{1}_{\{p<1 / 2\}}+v_{n+1}(x, p) \mathbf{1}_{\{p>1 / 2\}}
$$

and observe that $\int_{0}^{\infty} f_{n+1}(x, p) d x=1$ for all $p$.

Furthermore (see the proof of Corollary 2.1, or use Lemma 5.2 directly to calculate the Laplace transform of $f_{n+1}(x, p)$, or see [8], Chapter XIV, Section 6, formulas (6.6), (6.9)),

$$
f_{n+1}(x, p)=f_{1}^{*(n+1)}(x, p),
$$

where $^{6}$

$$
\begin{aligned}
f_{1}(x, p) & =\left(\frac{1-p}{p}\right) v_{1}(x, p) \mathbf{1}_{\{p<1 / 2\}}+v_{1}(x, p) \mathbf{1}_{\{p>1 / 2\}} \\
& =\left(\frac{1-p}{p}\right)^{1 / 2} e^{-x / p} I_{1}(2 x \sqrt{(1-p) / p}) x^{-1} \mathbf{1}_{\{p<1 / 2\}} \\
& +\left(\frac{p}{1-p}\right)^{1 / 2} e^{-x / p} I_{1}(2 x \sqrt{(1-p) / p}) x^{-1} \mathbf{1}_{\{p>1 / 2\}} \\
& +e^{-2 x} I_{1}(2 x) x^{-1} \mathbf{1}_{\{p=1 / 2\}} .
\end{aligned}
$$

The family $f_{1}(x, p), x \geqslant 0,0<p<1$, of the probability density functions (p.d.f.) corresponds to the random variables $\xi_{1}^{+}(p), p<1 / 2, \xi_{1}^{-}(p), p>1 / 2$, and $\xi_{1}^{\circ}$, which are non-negative and

$$
\begin{aligned}
\mathrm{P}\left\{\xi_{1}^{+}(p) \leqslant x\right\} & =\int_{0}^{x} f_{1}(y, p) d y, \quad p<1 / 2, \\
\mathrm{P}\left\{\xi_{1}^{-}(p) \leqslant x\right\} & =\int_{0}^{x} f_{1}(y, p) d y, \quad p>1 / 2, \\
\mathrm{P}\left\{\xi_{1}^{\circ} \leqslant x\right\} & =\int_{0}^{x} f_{1}(y, 1 / 2) d y .
\end{aligned}
$$

\footnotetext{
${ }^{6}$ That family is also known as the p.d.f. of the time of the first passage in a random walk process model, see Section 3.2 or [8], Chapter II, Section 7, formulas (7.13), (7.14) and [8], Chapter XIV, Section 6, formulas (6.6), (6.9).
} 
Moreover (compare (22) and (21)),

$$
\begin{aligned}
\varphi_{t}(u, p)=e^{-u \mu} \sum_{n \geqslant 0} \frac{(u \mu)^{n}}{n !}\left(\frac{p}{1-p}\right)^{n+1} \mathrm{P}\left\{\sum_{k=1}^{n+1} \xi_{k}^{+}(p) \leqslant \lambda t\right\} \mathbf{1}_{\{p<1 / 2\}} \\
+e^{-u \mu} \sum_{n \geqslant 0} \frac{(u \mu)^{n}}{n !} \mathrm{P}\left\{\sum_{k=1}^{n+1} \xi_{k}^{-}(p) \leqslant \lambda t\right\} \mathbf{1}_{\{p>1 / 2\}} \\
\quad+e^{-u \mu} \sum_{n \geqslant 0} \frac{(u \mu)^{n}}{n !} \mathrm{P}\left\{\sum_{k=1}^{n+1} \xi_{1}^{\circ} \leqslant \lambda t\right\} \mathbf{1}_{\{p=1 / 2\}},
\end{aligned}
$$

where each sum in brackets is composed of i.i.d. summands.

The Laplace transforms of the random variables $\xi_{1}^{+}(p)$ and $\xi_{1}^{-}(p)$ are $^{7}$

$$
\begin{aligned}
\gamma_{\xi_{1}^{+}(p)}(t) & =\frac{1+t p-\sqrt{(1+t p)^{2}-4 p(1-p)}}{2 p}, \quad p<1 / 2, \\
\gamma_{\xi_{1}^{-}(p)}(t) & =\frac{p}{1-p} \gamma_{\xi_{1}^{+}(p)}(t) \\
& =\frac{1+t p-\sqrt{(1+t p)^{2}-4 p(1-p)}}{2(1-p)}, \quad p>1 / 2,
\end{aligned}
$$

which is easy to check by direct calculation based on (58). Applying the arguments standard when the power moments are derived by use of the Laplace transform, i.e., $\mathrm{E} X^{k}=\left.(-1)^{k} \gamma_{X}^{(k)}(t)\right|_{t=0}$, one has in particular for the means of $\xi_{1}^{+}(p)$ and $\xi_{1}^{-}(p)$

$$
\mathrm{E} \xi_{1}^{+}(p)=\frac{p}{1-2 p}, \quad p<1 / 2, \quad \mathrm{E} \xi_{1}^{-}(p)=\frac{p}{2 p-1}, \quad p>1 / 2,
$$

for the second moments

$$
\mathrm{E}\left(\xi_{1}^{+}(p)\right)^{2}=\frac{2(1-p) p^{2}}{(1-2 p)^{3}}, \quad p<1 / 2, \quad \mathrm{E}\left(\xi_{1}^{-}(p)\right)^{2}=\frac{2 p^{3}}{(2 p-1)^{3}}, \quad p>1 / 2,
$$

for the third moments

$$
\mathrm{E}\left(\xi_{1}^{+}(p)\right)^{3}=\frac{6(1-p) p^{3}}{(1-2 p)^{5}}, \quad p<1 / 2, \quad \mathrm{E}\left(\xi_{1}^{-}(p)\right)^{3}=\frac{6 p^{4}}{(2 p-1)^{5}}, \quad p>1 / 2 .
$$

It is noteworthy that, unlike $\xi_{1}^{+}(p)$ and $\xi_{1}^{-}(p)$, the random variable $\xi_{1}^{\circ}$ has no power moments of order larger than $1 / 2$, since $I_{1}(x)=e^{x}(2 \pi x)^{-1 / 2}(1+\bar{o}(1))$, as $x \rightarrow \infty$, and both the normal and diffusion approximations, suggestive in (22) for $p>c>1 / 2$ and $p<c<1 / 2$, fail in a neighborhood of $p=1 / 2$. Being intuitive, this remark points a shortcoming immanent of these analytical methods rather than an irregularity of $\boldsymbol{\psi}_{t}(u, \tau)$ in the neighborhood of $p=1 / 2$.

3.2. Bessel functions and a random walk process. Suppose that a particle performs a random walk on the $x$-axis. Starting at $x=0$ in each step the particle moves either a unit distance to the right with probability $p$ or a unit distance to the left with probability $q(p+q=1,0<p<1)$. Suppose that the displacements of the particle occur at random times in the time interval $(0, \infty)$. Denote by $\nu(u)$ the number of steps taken in the interval $(0, u]$. We suppose that $\{\nu(u), 0 \leqslant u<\infty\}$

\footnotetext{
${ }^{7}$ The Laplace transform of the p.d.f. $f(x)=\sqrt{p / q} x^{-1} I_{1}(2 c \sqrt{p q} x) e^{-c x}, p \geqslant q$, is $\gamma(t)=$ $\left(c+t-\sqrt{(c+t)^{2}-4 c^{2} p q}\right) /(2 c q)$, see [8], Chapter XIV, Section 6, formulas (6.9) and (6.6).
} 
is a Poisson process of density $1 / p$ and that the successful displacements are independent of each other and independent of the process $\{\nu(u), 0 \leqslant u<\infty\}$. Denote by $\xi(u)$ the position of the particle at time $u$. In this case $\{\xi(u), 0 \leqslant u<\infty\}$ is a stochastic process having stationary independent increments, $\mathrm{P}\{\xi(0)=0\}=1$ and almost all sample functions of $\{\xi(u), 0 \leqslant u<\infty\}$ are step functions having jumps of magnitude 1 and -1 .

LEMMA 3.1 (Equations (3), (8) in $\S 22$ of [20]). In the random walk process model,

$$
\mathrm{P}\{\xi(u)=k\}=e^{-u / p}(p / q)^{k / 2} I_{k}(2 u \sqrt{q / p})=\frac{u}{k} v_{k}(u, p)
$$

for $k=0, \pm 1, \pm 2, \ldots$, and $d^{8}$

$$
\begin{aligned}
\mathrm{P}\left\{\sup _{0 \leqslant u \leqslant t} \xi(u)<k\right\}=1-k(p / q)^{k / 2} \int_{0}^{t} e^{-u / p} I_{k}(2 u \sqrt{q / p}) & \frac{d u}{u} \\
& =1-k \int_{0}^{t} \mathrm{P}\{\xi(u)=k\} \frac{d u}{u}=1-\int_{0}^{t} v_{k}(u, p) d u
\end{aligned}
$$

for $k>0$.

LEMMA 3.2 (Equation (9) in $\S 22$ of [20]). In the random walk process model,

$$
\mathrm{P}\left\{\sup _{0 \leqslant u \leqslant t} \xi(u)<k\right\}=\mathrm{P}\{\xi(t)<k\}-\left(\frac{p}{q}\right)^{k} \mathrm{P}\{\xi(t)<-k\}
$$

for $k>0$.

REMARK 3.1. Bearing in mind Lemma 3.1, Lemma 3.2 yields

$$
\begin{aligned}
k(p / q)^{k / 2} \int_{0}^{t} e^{-u / p} I_{k}(2 u \sqrt{q / p}) \frac{d u}{u}=e^{-t / p} & \sum_{i=k}^{\infty}(p / q)^{i / 2} I_{i}(2 t \sqrt{q / p}) \\
& +e^{-t / p} \sum_{i=k+1}^{\infty}(q / p)^{i / 2-k} I_{i}(2 t \sqrt{q / p}) .
\end{aligned}
$$

Denote by $\varsigma_{k}(p)$ the first hitting time of the point $k$ by the random walk process with the parameter $p \in(0,1)$. Evidently,

$$
\mathrm{P}\left\{\sup _{0 \leqslant u \leqslant t} \xi(u) \geqslant k\right\}=\int_{0}^{t} v_{k}(u, p) d u=\mathrm{P}\left\{\varsigma_{k}(p) \leqslant t\right\},
$$

so that $v_{k}(u, p), u \geqslant 0$, is the p.d.f. of the first hitting time of the point $k$ in the random walk process model (see also [8], Chapter II, Section 7 and Chapter XIV, Section 6 ). The densities $v_{k}(u, p), u \geqslant 0$, are defective for $p<1 / 2$ (random walk with drift to the left) and proper for $p \geqslant 1 / 2$ (random walk without drift, or with drift to the right).

3.3. Adaptive loading and rigid starting capital. Fundamental is the following result.

TheOREM 3.1. For $t, u>0$ fixed, $\varphi_{t}(u, p)$ regarded as a function of $p \in(0,1)$, is monotonously increasing, as $p$ increases.

\footnotetext{
${ }^{8}$ Note that $\mathrm{E} \xi(u)=[1-(q / p)] u, \mathrm{D} \xi(u)=u / p$.
} 


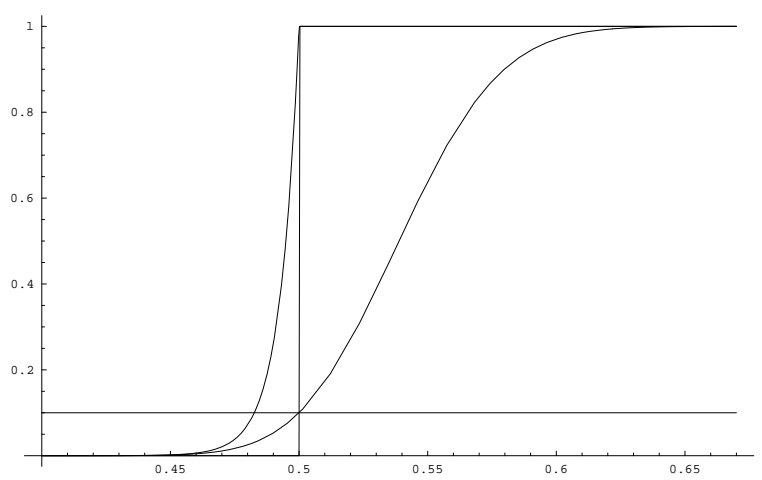

FiguRE 1. Graphs of $\varphi_{t}(u, p)$ with $t=200$ and of its upper bound $\varphi(u, p)$ regarded as functions of $p ; \lambda=1, \mu=1, u=33.2197$. The value of $\varphi_{t}(u, p)$ at $p=0.5$ (shown by vertical line) is 0.1 (shown by horizontal line). The value of $\varphi(u, p)$ at $p=0.5$ is 1 .

Proof. The proof is either analytical, by checking that $\frac{\partial}{\partial p} \varphi_{t}(u, p) \geqslant 0$, or probabilistic, by the application of the random walk process arguments. We present both to prepare the reader to more complicated set-up of Theorem 3.2.

The probabilistic arguments are as follows. Evidently, as $p_{1}<p_{2}$, the random time $\varsigma_{n+1}\left(p_{1}\right)$ is stochastically larger than $\varsigma_{n+1}\left(p_{2}\right)$. It yields

$$
\int_{0}^{\lambda t} v_{n+1}\left(x, p_{1}\right) d x=\mathrm{P}\left\{\varsigma_{n+1}\left(p_{1}\right) \leqslant \lambda t\right\} \leqslant \mathrm{P}\left\{\varsigma_{n+1}\left(p_{2}\right) \leqslant \lambda t\right\}=\int_{0}^{\lambda t} v_{n+1}\left(x, p_{2}\right) d x
$$

and the result follows directly from (19):

$$
\varphi_{t}(u, p)=e^{-u \mu} \sum_{n \geqslant 0} \frac{(u \mu)^{n}}{n !} \int_{0}^{\lambda t} v_{n+1}(x, p) d x .
$$

To apply the analytical arguments, i.e., to show that $\frac{\partial}{\partial p} \varphi_{t}(u, p) \geqslant 0$, note first that

$$
\frac{\partial}{\partial p} \varphi_{t}(u, p)=e^{-u \mu} \sum_{n \geqslant 0} \frac{(u \mu)^{n}}{n !} \int_{0}^{\lambda t} \frac{\partial}{\partial p} v_{n+1}(x, p) d x
$$

and

$$
\frac{\partial}{\partial p} v_{n+1}(x, p)=\frac{x}{p^{2}}\left\{v_{n+1}(x, p)-\frac{n+1}{n+2} v_{n+2}(x, p)\right\} .
$$

Indeed, bearing in mind (56), direct calculus yields

$$
\begin{aligned}
& \frac{\partial}{\partial p} v_{n+1}(x, p)=\left(\frac{p}{1-p}\right)^{\frac{n+2}{2}} \frac{n+1}{2 p^{2}} e^{-x / p}\left\{-I_{n}(2 x \sqrt{(1-p) / p})\right. \\
& \left.\quad+\frac{(n+1) p+2 x(1-p)}{x p^{1 / 2}(1-p)^{1 / 2}} I_{n+1}(2 x \sqrt{(1-p) / p})-I_{n+2}(2 x \sqrt{(1-p) / p})\right\}
\end{aligned}
$$

and the equality

$$
I_{n}(2 x \sqrt{(1-p) / p})=I_{n+2}(2 x \sqrt{(1-p) / p})+\frac{(n+1) \sqrt{p}}{x \sqrt{1-p}} I_{n+1}(2 x \sqrt{(1-p) / p}),
$$

which follows from (55), reduces (25) to (24) by means of some algebra. 
It is also noteworthy (see (24) and (20)) that

$$
\int_{0}^{\lambda t} \frac{\partial}{\partial p} v_{n+1}(x, p) d x=\frac{1}{p^{2}} \int_{0}^{\lambda t} x v_{n+1}(x, p) g_{n}(x, p) d x
$$

where

$$
g_{n}(x, p)=1-\left(\frac{p}{1-p}\right)^{1 / 2} r_{n+1}(2 x \sqrt{(1-p) / p})
$$

and

$$
r_{n+1}(2 x \sqrt{(1-p) / p})=I_{n+2}(2 x \sqrt{(1-p) / p}) / I_{n+1}(2 x \sqrt{(1-p) / p}) .
$$

Bearing in mind (23), (24) and (26), show that

$$
\int_{0}^{\lambda t} \frac{\partial}{\partial p} v_{n+1}(x, p) d x \geqslant 0
$$

for each integer $n \geqslant 0$ and $t \geqslant 0$; the left hand side of (27) is regarded as a function of $p \in(0,1)$.

For $p \leqslant 1 / 2, x \geqslant 0$ and $n \geqslant 0$ integer

$$
g_{n}(x, p) \geqslant 1-\left(\frac{p}{1-p}\right)^{1 / 2} \geqslant 0
$$

since $r_{n+1}(2 x \sqrt{(1-p) / p}) \leqslant 1$ by Lemma 5.3 . The integrand in the right hand side of (26) is therefore non-negative, which yields (27) immediately.

For $p>1 / 2$, note that

$$
\begin{gathered}
\int_{0}^{\infty} \frac{\partial}{\partial p} v_{n+1}(x, p) d x=\left(\frac{p}{1-p}\right)^{\frac{n+2}{2}} \frac{n+1}{p^{2}} \int_{0}^{\infty} e^{-x / p}\left[I_{n+1}(2 x \sqrt{(1-p) / p})\right. \\
\left.-\left(\frac{p}{1-p}\right)^{1 / 2} I_{n+2}(2 x \sqrt{(1-p) / p})\right] d x=\frac{1}{p^{2}} \int_{0}^{\infty} x v_{n+1}(x, p) g_{n}(x, p) d x=0 .
\end{gathered}
$$

It is yielded by the following lemma, which is a corollary of the equality (59) of Lemma 5.2 .

LEMMA 3.3. For integer $k \geqslant 1$ the following equality holds true:

$$
\int_{0}^{\infty} e^{-x / p} I_{k}(2 x \sqrt{(1-p) / p}) d x= \begin{cases}\left(\frac{1-p}{p}\right)^{k / 2}\left(\frac{p}{2 p-1}\right), & p>1 / 2, \\ \left(\frac{p}{1-p}\right)^{k / 2}\left(\frac{p}{1-2 p}\right), & p<1 / 2 .\end{cases}
$$

Consider $g_{n}(x, p)$. The theory of Bessel functions yields that $g_{n}(x, p)$, regarded as a function of $x \geqslant 0$, with $n$ and $p$ fixed, is continuous, differentiable and monotonously decreasing, as $x$ increases. For $p>1 / 2$, there exists $x^{*}=x^{*}(n, p)>0$ such that

$$
g_{n}(x, p) \begin{cases}\geqslant 0, & x \leqslant x^{*} \\ \leqslant 0, & x \geqslant x^{*}\end{cases}
$$

$g_{n}(0, p)=1$, and (evidently, $1-\sqrt{p /(1-p)}<0$ for $\left.p>1 / 2\right)$

$$
g_{n}(x, p) \rightarrow 1-\sqrt{p /(1-p)}, \quad \text { as } x \rightarrow \infty .
$$

It is easy to demonstrate that $x_{2}=x_{2}(n, p) \leqslant x^{*} \leqslant x_{1}(n, p)=x_{1}$, where

$$
x_{1}=\frac{\left(n+\frac{3}{2}\right) p}{2 p-1}, \quad x_{2}=\frac{\left(n+\frac{3}{2}\right) p}{2(2 p-1)}+\frac{\sqrt{\left(4 n^{2} p+4 n(7 p-2)+41 p-16\right) p}}{4(2 p-1)} .
$$




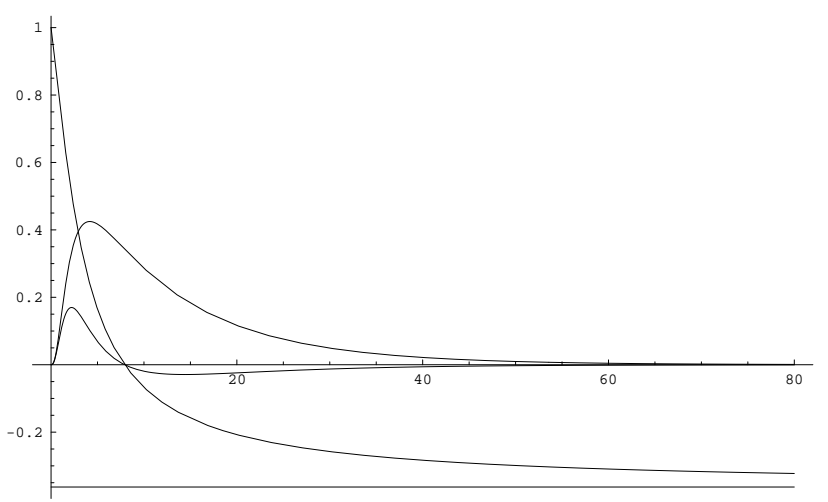

Figure 2. Functions $g_{n}(x, p), x v_{n+1}(x, p)$ and $x v_{n+1}(x, p) g_{n}(x, p)$ for $p=0.65, n=2$. The asymptote for $g_{n}(x, p)$, as $x \rightarrow \infty$, is $1-\sqrt{p /(1-p)}=-0.36277$.

To show it, apply Lemma 5.3 which yields

$$
\begin{aligned}
1-\frac{2 x}{n+\frac{3}{2}+\sqrt{4 x^{2}(1-p) / p+\left(n+\frac{3}{2}\right)^{2}}} & \leqslant g_{n}(x, p) \\
\leqslant 1 & -\frac{2 x}{n+\frac{3}{2}+\sqrt{4 x^{2}(1-p) / p+\left(n+\frac{5}{2}\right)^{2}}},
\end{aligned}
$$

and $x_{1}$ and $x_{2}$ are the positive solutions of the quadratic equations

$$
4 x^{2}(1-p) / p+(n+3 / 2)^{2}=(2 x-n-3 / 2)^{2}
$$

and

$$
4 x^{2}(1-p) / p+(n+5 / 2)^{2}=(2 x-n-3 / 2)^{2},
$$

respectively.

Infer from (29) that the "positive mass" in the integral $\int_{0}^{\infty} x v_{n+1}(x, p) g_{n}(x, p) d x$ (which equals to zero, see $(28)$ ) is concentrated in the interval $\left(0, x^{*}\right)$, and the "negative mass" is concentrated in the interval $\left(x^{*}, \infty\right)$; by $(28)$, these masses are equal. Switching to $\int_{0}^{\lambda t} x v_{n+1}(x, p) g_{n}(x, p) d x$, i.e., cutting the mass at the right of the level $\lambda t$ in $\int_{0}^{\infty} x v_{n+1}(x, p) g_{n}(x, p) d x$, we deduct a negative mass from the total mass zero, whichever $\lambda t$ be, and the rest (i.e., $\int_{0}^{\lambda t} x v_{n+1}(x, p) g_{n}(x, p) d x=$ $p^{2} \int_{0}^{\lambda t} \frac{\partial}{\partial p} v_{n+1}(x, p) d x$, see (26)) must be positive. It completes the proof.

REMARK 3.2. Since $p(\tau)=1 /(2+\tau)\left(\right.$ see $(18)$ and note that $\left.\frac{\partial p(\tau)}{\partial \tau}=-p^{2}(\tau)\right)$, application of the chain rule for differentiation of composite functions

$$
\frac{\partial}{\partial \tau} \boldsymbol{\psi}_{t}(u, \tau)=\left.\frac{\partial}{\partial p} \varphi_{t}(u, p)\right|_{p=p(\tau)} \cdot \frac{\partial p(\tau)}{\partial \tau}
$$

yields immediately that the probability $\boldsymbol{\psi}_{t}(u, \tau)$, regarded as a function of loading $\tau \in(-1, \infty)$, is monotonously decreasing, as $\tau$ increases. 
3.4. Adaptive loading and adaptive starting capital. Generalize the framework of Section 3.2 and consider the starting capital $u_{p}$ dependent on $p$ in a rather specific way. The nature of this dependence is becoming clear in the proof of Theorem 4.2 .

TheOREM 3.2. For $u_{p}=u+\lambda t(2 p-1) /(\mu p)$ and $t, u>0$ fixed, $\varphi_{t}(u, p)$ regarded as a function of $p \in(0,1)$, is monotonously decreasing, as $p$ increases.



FiguRE 3. Graphs of $\varphi_{t}\left(u_{p}, p\right)$ with $t=200$ and of its upper bound $\varphi\left(u_{p}, p\right)$ regarded as functions of $p ; \lambda=1, \mu=1, u=33.2197$. The value of $\varphi_{t}\left(u_{p}, p\right)$ at $p=0.5$ is 0.1 (note that $u_{1 / 2}=u$ ). The value of $\varphi\left(u_{p}, p\right)$ at $p=0.5$ is 1 .

ProOF. For brevity sake ${ }^{9}$, set $\Pi_{n}(p)=e^{-u_{p} \mu} \frac{\left(u_{p} \mu\right)^{n}}{n !}, n \geqslant 0$, and recall that $\varphi_{t}\left(u_{p}, p\right)=\sum_{n \geqslant 0} \Pi_{n}(p) \int_{0}^{\lambda t} v_{n+1}(x, p) d x$. Prove that for each $t>0$ and $u>0$ fixed

Note first that

$$
\frac{\partial}{\partial p} \varphi_{t}\left(u_{p}, p\right) \leqslant 0
$$

$$
\frac{\partial}{\partial p} \varphi_{t}\left(u_{p}, p\right)=\sum_{n \geqslant 0} \Pi_{n}(p) \vartheta_{n+1}(p ; \lambda t)
$$

where

$$
\begin{aligned}
\vartheta_{n+1}(p ; \lambda t)=-\frac{\lambda t}{p^{2}} \int_{0}^{\lambda t}\left(v_{n+1}(x, p)-v_{n+2}(x, p)\right) d x & \\
& +\frac{1}{p^{2}} \int_{0}^{\lambda t} x\left(v_{n+1}(x, p)-\frac{n+1}{n+2} v_{n+2}(x, p)\right) d x
\end{aligned}
$$

Indeed,

$$
\frac{\partial}{\partial p} \varphi_{t}\left(u_{p}, p\right)=\sum_{n \geqslant 0}\left(\frac{\partial}{\partial p} \Pi_{n}(p)\right) \int_{0}^{\lambda t} v_{n+1}(x, p) d x+\sum_{n \geqslant 0} \Pi_{n}(p) \int_{0}^{\lambda t} \frac{\partial}{\partial p} v_{n+1}(x, p) d x
$$

\footnotetext{
${ }^{9}$ Observe that $\Pi_{n}(1 / 2)=e^{-u \mu}(u \mu)^{n} / n !$, and hence $\varphi_{t}\left(u_{1 / 2}, 1 / 2\right)=\varphi_{t}(u, 1 / 2)$.
} 
and

$$
\frac{\partial}{\partial p} \Pi_{0}(p)=-\frac{\lambda t}{p^{2}} \Pi_{0}(p), \quad \frac{\partial}{\partial p} \Pi_{n}(p)=-\frac{\lambda t}{p^{2}}\left(\Pi_{n}(p)-\Pi_{n-1}(p)\right), \quad n \geqslant 1 .
$$

The latter is checked by direct differentiation.

Integrating by parts, it is easily seen that (32) may be rewritten as

$$
\begin{aligned}
\vartheta_{n+1}(p ; \lambda t)=\frac{1}{p^{2}} \int_{0}^{\lambda t} \frac{x}{n+2} v_{n+2}(x, p) & d x \\
& -\frac{1}{p^{2}} \int_{0}^{\lambda t}\left[V_{n+1}(x, p)-V_{n+2}(x, p)\right] d x,
\end{aligned}
$$

where $V_{k}(x, p)=\int_{0}^{x} v_{k}(z, p) d z ;$ for notational simplicity introduce

$$
d_{n}(x, p)=\frac{x}{n+2} v_{n+2}(x, p)-\left[V_{n+1}(x, p)-V_{n+2}(x, p)\right] .
$$

For $p \leqslant 1 / 2$, the inequality $\vartheta_{n+1}(p ; \lambda t)=\frac{1}{p^{2}} \int_{0}^{\lambda t} d_{n}(x, p) d x \leqslant 0$ is straightforward. Indeed, one should merely observe that, by Lemma 3.1,

$V_{n+1}(x, p)-V_{n+2}(x, p)=\mathrm{P}\left\{\sup _{0 \leqslant z \leqslant x} \xi(z)=n+1\right\}, \frac{x}{n+2} v_{n+2}(x, p)=\mathrm{P}\{\xi(x)=n+2\}$

for any $x \geqslant 0$ and integer $n \geqslant 0$, and that

$$
\mathrm{P}\left\{\sup _{0 \leqslant z \leqslant x} \xi(z)=n+1\right\} \geqslant \mathrm{P}\{\xi(x)=n+2\}
$$

for $p \leqslant 1 / 2$. The latter can be easily seen by using the reflection principle: for $p=1 / 2$, the trajectories for which $\xi(x)=n+2$ are in the one-to-one correspondence (by means of reflection with respect to the barrier $n+1$ ) with those for which $\xi(x)=n$ and $\sup _{0 \leqslant z \leqslant x} \xi(z)=n+1$; the number of these trajectories is less than the total number of trajectories for which $\sup _{0 \leqslant z \leqslant x} \xi(z)=n+1$. Evidently, that argument grows even stronger for $p<1 / 2$.

For $p>1 / 2$, prove first that

$$
\vartheta_{n+1}(p ; \infty)=\frac{1}{p^{2}} \int_{0}^{\infty} d_{n}(x, p) d x=0 .
$$

By Lemma 3.3, for $p>1 / 2$,

$$
\begin{gathered}
\int_{0}^{\infty}\left[V_{n+1}(x, p)-V_{n+2}(x, p)\right] d x \\
=\left.x\left[V_{n+1}(x, p)-V_{n+2}(x, p)\right]\right|_{0} ^{\infty}-\int_{0}^{\infty} x d\left[V_{n+1}(x, p)-V_{n+2}(x, p)\right] \\
=\int_{0}^{\infty} x\left[v_{n+2}(x, p)-v_{n+1}(x, p)\right] d x \\
=\left(\frac{p}{1-p}\right)^{\frac{n+2}{2}}(n+2) \int_{0}^{\infty} e^{-x / p} I_{n+2}(2 x \sqrt{(1-p) / p}) d x \\
-\left(\frac{p}{1-p}\right)^{\frac{n+1}{2}}(n+1) \int_{0}^{\infty} e^{-x / p} I_{n+1}(2 x \sqrt{(1-p) / p}) d x \\
=\frac{p}{2 p-1}(n+2)-\frac{p}{2 p-1}(n+1)=\frac{p}{2 p-1}
\end{gathered}
$$


and

$$
\begin{array}{r}
\int_{0}^{\infty} \frac{x}{n+2} v_{n+2}(x, p) d x=\left(\frac{p}{1-p}\right)^{\frac{n+2}{2}} \int_{0}^{\infty} e^{-x / p} I_{n+2}(2 x \sqrt{(1-p) / p}) d x \\
=\frac{p}{2 p-1}
\end{array}
$$

which yields (35).

Consider the integrand $d_{n}(x, p)$ in (35). The theory of Bessel functions yields that $d_{n}(x, p)$, regarded as a function of $x \geqslant 0$, with $n$ and $p$ fixed, is continuous and differentiable. For $p>1 / 2$, there exists $x^{*}>0$ such that

$$
\begin{gathered}
d_{n}(x, p)=\frac{x}{n+2} v_{n+2}(x, p)-\left[V_{n+1}(x, p)-V_{n+2}(x, p)\right] \begin{cases}\leqslant 0, & x \leqslant x^{*}, \\
\geqslant 0, & x \geqslant x^{*},\end{cases} \\
d_{n}(0, p)=0 \text {, and } \quad d_{n}(x, p) \rightarrow 0, \quad \text { as } x \rightarrow \infty .
\end{gathered}
$$

To prove $(37)$, recall that

$$
\begin{aligned}
d_{n}(x, p)= & \left(\frac{p}{1-p}\right)^{\frac{n+2}{2}} e^{-x / p} I_{n+2}(2 x \sqrt{(1-p) / p}) \\
& -\left(\frac{p}{1-p}\right)^{\frac{n+1}{2}}(n+1) \int_{0}^{x} e^{-z / p} I_{n+1}(2 z \sqrt{(1-p) / p}) \frac{d z}{z} \\
& +\left(\frac{p}{1-p}\right)^{\frac{n+2}{2}}(n+2) \int_{0}^{x} e^{-z / p} I_{n+2}(2 z \sqrt{(1-p) / p}) \frac{d z}{z}
\end{aligned}
$$

and apply the next lemma which is a corollary of the equality (58) of Lemma 5.2.

LEMma 3.4. For integer $k \geqslant 1$ the following equality holds true:

$$
\int_{0}^{\infty} e^{-x / p} I_{k}(2 x \sqrt{(1-p) / p}) \frac{d x}{x}= \begin{cases}\frac{1}{k}\left(\frac{1-p}{p}\right)^{k / 2}, & p>1 / 2 \\ \frac{1}{k}\left(\frac{p}{1-p}\right)^{k / 2}, & p<1 / 2 .\end{cases}
$$

Evidently, by Lemma 3.4,

$$
d_{n}(x, p) \rightarrow 0-1+1=0, \quad \text { as } x \rightarrow \infty .
$$

To check relation (36), note (using (55) and (56)) that

$$
\begin{aligned}
\frac{\partial}{\partial x} d_{n}(x, p)=\frac{x}{(n+1)} & \sqrt{p(1-p)} \\
\times & \left\{2 \sqrt{p(1-p)}\left(1-\frac{n+1}{2 x}\right)-r_{n+1}(2 x \sqrt{(1-p) / p})\right\}
\end{aligned}
$$

where the first factor is non-negative for any $x \geqslant 0$, the factor in brackets has exactly two positive zeros, $x_{1}=x_{1}(n, p)$ and $x_{2}=x_{2}(n, p)\left(x_{1}<x_{2}\right)$, being negative for $0 \leqslant x \leqslant x_{1}$, positive for $x_{1} \leqslant x \leqslant x_{2}$, and negative for $x \geqslant x_{2}$.

To prove that the equation

$$
2 \sqrt{p(1-p)}\left(1-\frac{n+1}{2 x}\right)=r_{n+1}(2 x \sqrt{(1-p) / p})
$$

has exactly two positive roots, note that both sides of (41) are monotonously increasing functions of $x$; the left hand side of (41) denoted by $l_{n, p}(x)$ equals 




FigURE 4 . Functions $d_{n}(x, p)$ and $\frac{\partial}{\partial x} d_{n}(x, p)$ for $p=0.65, n=2$; zeros of $\frac{\partial}{\partial x} d_{n}(x, p)$ are points of inflection of $d_{n}(x, p)$.

to $-\infty$ at the origin; the right hand side of (41) equals to zero at the origin; $r_{n+1}(2 x \sqrt{(1-p) / p}) \rightarrow 1$, as $x \rightarrow \infty ; l_{n, p}(x) \rightarrow 2 \sqrt{p(1-p)}$, as $x \rightarrow \infty$, and $2 \sqrt{p(1-p)}<1$ for $p>1 / 2$.

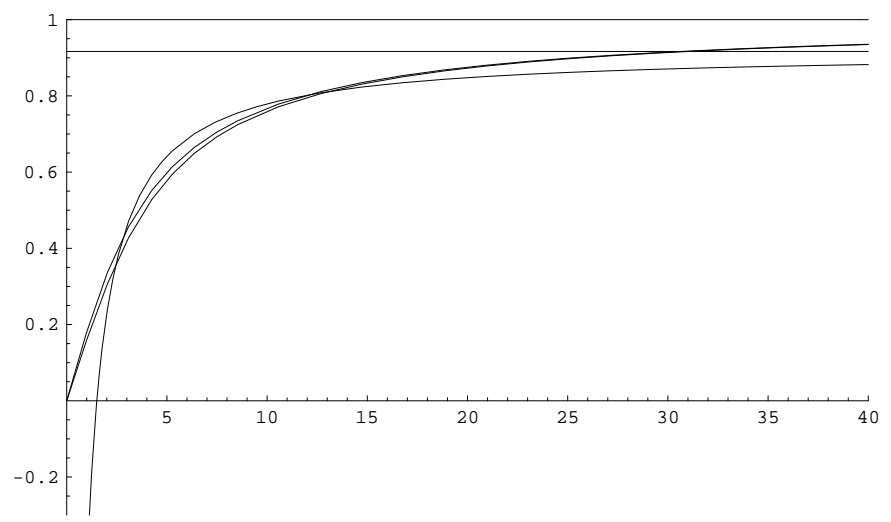

Figure 5. Left and right hand sides of equation (41) and upper bound (42) for the latter, $p=0.7, n=2 ; x_{1}=2.47697, \bar{x}_{1}=$ 2.83409, $x_{2}=12.0409, \bar{x}_{2}=13.0892$. Two horizontal lines are the asymptotes $2 \sqrt{p(1-p)}=0.91651$ and 1 .

To check that $l_{n, p}(x)$ and $r_{n+1}(2 x \sqrt{(1-p) / p})$ intersect twice for $p>1 / 2$, it is sufficient to verify that $l_{n, p}(x)$ and the upper bound of $r_{n+1}(2 x \sqrt{(1-p) / p})$ given 
by (the lower and upper bounds (42) follow from Lemma 5.3)

$$
\begin{aligned}
\frac{2 x \sqrt{(1-p) / p}}{n+\frac{3}{2}+\sqrt{4 x^{2}(1-p) / p+\left(n+\frac{5}{2}\right)^{2}}} & \leqslant r_{n+1}(2 x \sqrt{(1-p) / p}) \\
& \leqslant \frac{2 x \sqrt{(1-p) / p}}{n+\frac{3}{2}+\sqrt{4 x^{2}(1-p) / p+\left(n+\frac{3}{2}\right)^{2}}}
\end{aligned}
$$

intersect twice. It is noteworthy that both bounds (42) are very sharp.

Applying the upper bound (42), which is obviously a monotonously increasing function of $x$, consider the equation

$$
2 \sqrt{p(1-p)}\left(1-\frac{n+1}{2 x}\right)=\frac{2 x \sqrt{(1-p) / p}}{n+\frac{3}{2}+\sqrt{4 x^{2}(1-p) / p+\left(n+\frac{3}{2}\right)^{2}}} .
$$

By means of direct algebra it rewrites as the quadratic equation

$$
x^{2}+2 a_{p, n} x+b_{p, n}=0
$$

with the coefficients

$$
a_{p, n}=p \frac{n+\frac{3}{2}-2(1-p)(n+1)}{4 p(1-p)-1}, \quad b_{p, n}=p(n+1) \frac{(1-p)(n+1)-\left(n+\frac{3}{2}\right)}{4 p(1-p)-1} .
$$

Note that for $n \geqslant 0$ integer and $p \in(1 / 2,1)$

$$
\begin{gathered}
a_{p, n}=-p \frac{2 n(2 p-1)+(4 p-1)}{2(1-2 p)^{2}} \leqslant 0, \quad b_{p, n}=p(n+1) \frac{1+2 p(n+1)}{2(1-2 p)^{2}} \geqslant 0, \\
a_{p, n}^{2}-b_{p, n}=p \frac{n(4 p-2)+5 p-2}{4(1-2 p)^{4}} \geqslant 0, \quad b_{p, n}+a_{p, n}=p \frac{2+3 n+2 p\left(n^{2}-1\right)}{2(1-2 p)^{2}} \geqslant 0,
\end{gathered}
$$

so that $0<-a_{p, n} \leqslant b_{p, n}$ and $a_{p, n}^{2} \geqslant b_{p, n}$. It yields that the equation (43) has two positive roots,

$$
\bar{x}_{2}(p, n)=-a_{p, n}+\sqrt{a_{p, n}^{2}-b_{p, n}}, \quad \bar{x}_{1}(p, n)=-a_{p, n}-\sqrt{a_{p, n}^{2}-b_{p, n}} .
$$

Plainly, $x_{1}<\bar{x}_{1}<x_{2}<\bar{x}_{2}$. Applying the lower bound (42) in the similar way, one will get the lower bounds for $x_{1}$ and $x_{2}$.

Infer from (36) that the "positive mass" in the integral $\int_{0}^{\infty} d_{n}(x, p) d x$ (which equals to zero, see (35)) is concentrated in the interval $\left(x^{*}, \infty\right)$, and the "negative mass" is concentrated in the interval $\left(0, x^{*}\right)$; by $(35)$, these masses are equal. Switching to $\int_{0}^{\lambda t} d_{n}(x, p) d x$, i.e., cutting the mass at the right of the level $\lambda t$ in $\int_{0}^{\infty} d_{n}(x, p) d x$, we deduct a positive mass from the total mass zero, whichever $\lambda t$ be, and the rest (i.e., $\int_{0}^{\lambda t} d_{n}(x, p) d x=p^{2} \vartheta_{n+1}(p ; \lambda t)$, see $\left.(33)\right)$ must be negative. It completes the proof.

REMARK 3.3. Since $p(\tau)=1 /(2+\tau)$ (see (18) and note that $\frac{\partial p(\tau)}{\partial \tau}=-p^{2}(\tau)$, $\left.u_{p(\tau)}=u-\frac{\lambda t}{\mu} \tau\right)$, application of the chain rule for differentiation of composite functions

$$
\frac{\partial}{\partial \tau} \boldsymbol{\psi}_{t}\left(u_{p(\tau)}, \tau\right)=\left.\frac{\partial}{\partial p} \varphi_{t}\left(u_{p}, p\right)\right|_{p=p(\tau)} \cdot \frac{\partial p(\tau)}{\partial \tau}
$$

yields immediately that $\boldsymbol{\psi}_{t}\left(u_{p(\tau)}, \tau\right)$, regarded as a function of loading $\tau \in(-1, \infty)$, is monotonously increasing, as $\tau$ increases. 


\section{Two control strategies}

Definition 4.1. The target value $u_{\lambda, \mu}(\alpha, t)$ of the risk reserve corresponding to a level $\alpha \in(0,1)$ is the value $u=u_{\lambda, \mu}(\alpha, t)$ which satisfies the equation

$$
\psi_{t}(u ; 0)=\alpha .
$$

THEOREM 4.1. In the classical risk model the target value $u_{\lambda, \mu}(\alpha, t)$ of the risk reserve corresponding to the prescribed value $\alpha \in(0,1)$ of the probability of ruin is the solution of the equation

$$
\int_{0}^{\pi} f_{t}(x ; u, 0) d x=\pi(1-\alpha)
$$

where $f_{t}(x ; u, 0)=(2(1-\cos x))^{-1} \exp \{(\cos x-1)(u \mu+2 \lambda t)\}[\cos (u \mu \sin x)-$ $\cos (u \mu \sin x+2 x)]$.

Proof. Bearing in mind (10), equation (45) easily rewrites as (46).

Numerical calculations yield the following results.

TABLE 4.1. Solutions of (46) for $\mu=\lambda=1$.

\begin{tabular}{c|cccc}
\hline$t$ & $\alpha=0.1$ & $\alpha=0.01$ & $\alpha=0.001$ & $\alpha=0.0001$ \\
\hline \hline 50 & 16.7422 & 27.9872 & 37.2083 & 45.3298 \\
100 & 23.5722 & 38.6811 & 50.8686 & 61.4894 \\
150 & 28.8077 & 46.8789 & 61.3408 & 73.8748 \\
200 & 33.2197 & 53.7878 & 70.1593 & 84.3084 \\
\hline
\end{tabular}

4.1. Control without borrowing. Set (see equation (1) and discussion in Section 1)

$$
u_{z, t}=u_{\lambda, \mu}(\alpha, t)+z \quad \text { and } \quad \tau_{z, t}=-\frac{\mu}{\lambda t} z
$$

and introduce

$$
\alpha(z)=\frac{\lambda t}{\lambda t-\mu z} \exp \left\{\frac{\mu^{2} z\left(u_{\lambda, \mu}(\alpha, t)+z\right)}{\lambda t-\mu z}\right\} .
$$

Put

$$
z^{*}=\frac{\lambda t}{\mu}-\frac{1}{2 \mu}-\frac{\lambda t}{\mu}\left(1+\frac{\mu u_{\lambda, \mu}(\alpha, t)}{\lambda t}+\frac{1}{4 \lambda^{2} t^{2}}\right)^{1 / 2} .
$$

Applying the expansion $(1+x)^{1 / 2}=1+x / 2+\ldots$ in the neighborhood of zero, note that for large $t$ and small $u_{\lambda, \mu}(\alpha, t) t^{-1}$ (see $[\mathbf{1 5}]$ where $u_{\lambda, \mu}(\alpha, t)$ is proved to be $c \sqrt{t}(1+\bar{o}(1))$ with explicitly written constant $c$, as $t \rightarrow \infty)$

$$
z^{*}=-\frac{u_{\lambda, \mu}(\alpha, t)+1}{2}-\frac{1}{8 \mu \lambda t}+\ldots
$$

THEOREM 4.2. For $z \in[a, b],-u_{\lambda, \mu}(\alpha, t)<a<0<b<\frac{\lambda}{\mu} t$, and for the control (47) the probability $\boldsymbol{\psi}_{t}\left(u_{z, t}, \tau_{z, t}\right)$, regarded as a function of $z$, is monotonously decreasing, as $z$ increases, and

$$
\boldsymbol{\psi}_{t}\left(u_{z, t}, \tau_{z, t}\right) \leqslant \alpha(z) \mathbf{1}_{\left\{z<z^{*}\right\}}+\alpha\left(z^{*}\right) \mathbf{1}_{\left\{z>z^{*}\right\}} .
$$


ProOF. Developing Remark 3.3, note first that the probability $\boldsymbol{\psi}_{t}\left(u_{z, t}, \tau_{z, t}\right)$, regarded as a function of $z \in[a, b],-u_{\lambda, \mu}(\alpha, t)<a<0<b<\frac{\lambda}{\mu} t$, is monotonously decreasing, as $z$ increases. To prove it, write

$$
\psi_{t}\left(u_{z, t}, \tau_{z, t}\right)=\varphi_{t}\left(u_{p\left(\tau_{z, t}\right)}, p\left(\tau_{z, t}\right)\right),
$$

where $u_{p}=u_{\lambda, \mu}(\alpha, t)+\lambda t(2 p-1) /(\mu p), p(\tau)=1 /(2+\tau)$; naturally, $u_{p\left(\tau_{z, t}\right)}=$ $u_{\lambda, \mu}(\alpha, t)+z=u_{z, t}$. By the chain rule for differentiation of composite functions,

$$
\frac{\partial}{\partial z} \boldsymbol{\psi}_{t}\left(u_{z, t}, \tau_{z, t}\right)=\left.\left.\frac{\partial}{\partial p} \varphi_{t}\left(u_{p}, p\right)\right|_{p=p\left(\tau_{z, t}\right)} \cdot \frac{\partial p(\tau)}{\partial \tau}\right|_{\tau=\tau_{z, t}} \cdot \frac{\partial \tau_{z, t}}{\partial z}
$$

Since

$$
\frac{\partial p(\tau)}{\partial \tau}=-p^{2}(\tau) \leqslant 0, \quad \frac{\partial \tau_{z, t}}{\partial z}=-\frac{\mu}{\lambda t} \leqslant 0,
$$

the result follows from Theorem 3.2.

Furthermore,

$$
\boldsymbol{\psi}_{t}\left(u_{z, t}, \tau_{z, t}\right)=\varphi_{t}\left(u_{p\left(\tau_{z, t}\right)}, p\left(\tau_{z, t}\right)\right) \leqslant \varphi\left(u_{p\left(\tau_{z, t}\right)}, p\left(\tau_{z, t}\right)\right)
$$

and (see $(21))$

$$
\varphi(u, p)=\frac{p}{1-p} \exp \left\{-u \mu \frac{1-2 p}{1-p}\right\} \mathbf{1}_{\{p<1 / 2\}}+\mathbf{1}_{\{p \geqslant 1 / 2\}} .
$$

Put $u_{p\left(\tau_{z, t}\right)}=u_{\lambda, \mu}(\alpha, t)+z$ and $p\left(\tau_{z, t}\right)=\lambda t /(2 \lambda t-\mu z)$ into (51). The result follows from minimization of the upper bound in (50)-(51) for $p\left(\tau_{z, t}\right)<1 / 2$. The latter inequality is equivalent to $z<0$.

The bound of Theorem 4.2 is illustrated by Fig. 6 : in the point $z^{*}$ that bowlshaped upper bound for $\boldsymbol{\psi}_{t}\left(u_{z, t}, \tau_{z, t}\right)$ achieves its minimum.

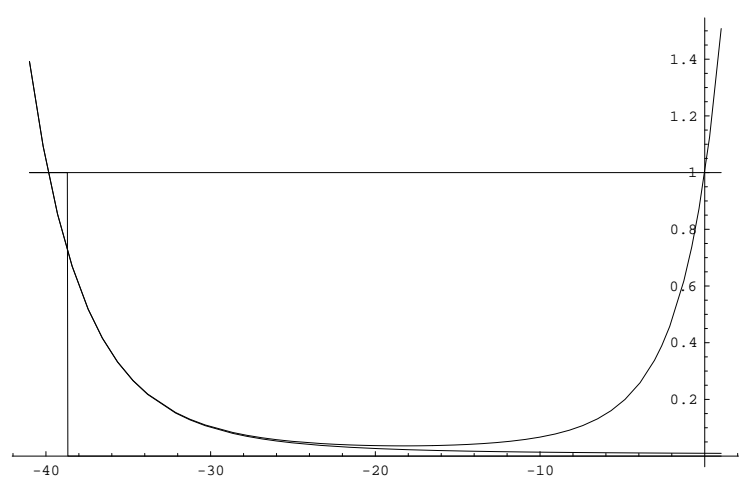

Figure 6 . Finite time $(t=200, \mu=\lambda=1)$ ruin probability $\boldsymbol{\psi}_{t}\left(u_{z, t}, \tau_{z, t}\right)$ and its upper bound $\varphi\left(u_{p\left(\tau_{z, t}\right)}, p\left(\tau_{z, t}\right)\right)$ regarded as functions of the capital deviation $z ; u_{z, t}=u_{\lambda, \mu}(\alpha, t)+z, \tau_{z, t}=$ $-\frac{\mu}{\lambda t} z$, target value is $u_{\lambda, \mu}(\alpha, t)=38.6811$ (vertical line shows the value $-u_{\lambda, \mu}(\alpha, t)$ bounding the range of $z$ from below) and the prescribed value of the probability of ruin is $\alpha=0.01$.

REMARK 4.1. For the strategy (47), the upper bound on $\mathrm{P}\{$ ruin within $n$ years $\}$ in (3) follows directly from equation (4), inequality (16) and Theorem 4.2. 
4.2. Control with borrowing. Set

$$
\bar{u}_{z, t}=\left\{\begin{array}{ll}
u_{\lambda, \mu}(\alpha, t), & z \leqslant 0, \\
u_{\lambda, \mu}(\alpha, t)+z, & z>0
\end{array} \quad \text { and } \quad \tau_{z, t}=-\frac{\mu}{\lambda t} z\right.
$$

This choice means starting from the past-year-end risk reserve when $z$ is surplus over $u_{\lambda, \mu}(\alpha, t)$, and borrowing when $z$ is deficit under $u_{\lambda, \mu}(\alpha, t)$. The adaptive loading $\tau_{z, t}$ is taken in the same way as in (47), i.e., positive in case of deficit and negative in case of surplus.

It is noteworthy that in case of deficit $(z<0)$ the sum borrowed at the beginning of the forthcoming insurance year is $|z|$ and, bearing in mind Theorem 2.2,

$$
\mathrm{E} R_{t}\left(\bar{u}_{z, t}, \tau_{z, t}\right)= \begin{cases}u_{\lambda, \mu}(\alpha, t)-z, & z \leqslant 0 \\ u_{\lambda, \mu}(\alpha, t), & z>0\end{cases}
$$

So, the sum borrowed amounts exactly to the average surplus at the end of the year, which makes possible to reimburse the borrowing at the end of the year.

Note also that we disregard borrowing costs for simplicity. But even that simplified set-up is sensible. In practice, a large corporation comprises different business branches (insurance, investment, banking, etc.) and may redistribute assets, if necessary.

TheOREM 4.3. For $z \in[a, b],-u_{\lambda, \mu}(\alpha, t)<a<0<b<\frac{\lambda}{\mu} t$, and for the control (52)

$$
\boldsymbol{\psi}_{t}\left(\bar{u}_{z, t}, \tau_{z, t}\right) \leqslant \alpha
$$

PROOF. It was just shown in the proof of Theorem 4.2 that the probability $\psi_{t}\left(u_{\lambda, \mu}(\alpha, t)+z, \tau_{z, t}\right)$ regarded as a function of $z \in[a, b],-u_{\lambda, \mu}(\alpha, t)<a<0<$ $b<\frac{\lambda}{\mu} t$, is monotonously decreasing, as $z$ increases. That result was derived from (48), (49) and Theorem 3.2. The similar arguments, applying the chain rule for differentiation of composite functions

$$
\frac{\partial}{\partial z} \psi_{t}\left(u_{\lambda, \mu}(\alpha, t), \tau_{z, t}\right)=\left.\left.\frac{\partial}{\partial p} \varphi_{t}\left(u_{\lambda, \mu}(\alpha, t), p\right)\right|_{p=p\left(\tau_{z, t}\right)} \cdot \frac{\partial p(\tau)}{\partial \tau}\right|_{\tau=\tau_{z, t}} \cdot \frac{\partial \tau_{z, t}}{\partial z}
$$

inequalities (49) and Theorem 3.1 assert that the probability $\psi_{t}\left(u_{\lambda, \mu}(\alpha, t), \tau_{z, t}\right)$ regarded as a function of $z \in[a, b],-u_{\lambda, \mu}(\alpha, t)<a<0<b<\frac{\lambda}{\mu} t$, is monotonously increasing, as $z$ increases.

The proof finally comes from that

$$
\left.\boldsymbol{\psi}_{t}\left(u_{\lambda, \mu}(\alpha, t), \tau_{z, t}\right)\right|_{z=0}=\left.\boldsymbol{\psi}_{t}\left(u_{\lambda, \mu}(\alpha, t)+z, \tau_{z, t}\right)\right|_{z=0}=\alpha
$$

The assertion of Theorem 4.3 is illustrated by Fig. 7 .

REMARK 4.2. For the strategy (52), the upper bound on $\mathrm{P}\{$ ruin within $n$ years $\}$ in (3) follows directly from equation (4), inequality (16) and Theorem 4.3. For that strategy the bound appears very simple: $\mathrm{P}\{$ ruin within $n$ years $\} \leqslant n \alpha$. 


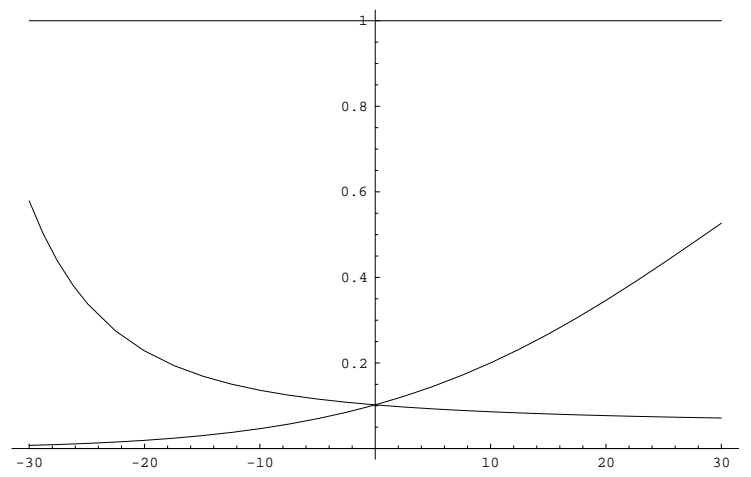

Figure 7. Two finite time $(t=200, \mu=\lambda=1)$ ruin probabilities $\boldsymbol{\psi}_{t}\left(u_{\lambda, \mu}(\alpha, t)+z, \tau_{z, t}\right)$ (decreasing graph) and $\boldsymbol{\psi}_{t}\left(u_{\lambda, \mu}(\alpha, t), \tau_{z, t}\right)$ (increasing graph) regarded as functions of the capital deviation $z ; \tau_{z, t}=-\frac{\mu}{\lambda t} z$, target value is $u_{\lambda, \mu}(\alpha, t)=33.2197$, the prescribed value of the probability of ruin is $\alpha=0.1$.

\section{Auxiliary results}

For $z>0$ and $n=0,1,2, \ldots$, the modified Bessel function of $n$-th order (see fundamental treatise [22], or [23], Chapter XVII, 17.7, or [1]) is ${ }^{10}$

$$
I_{n}(z)=\sum_{k=0}^{\infty} \frac{1}{k !(n+k) !}\left(\frac{z}{2}\right)^{n+2 k} .
$$

For $n$ integer, the integral representation

$$
I_{n}(z)=\frac{1}{\pi} \int_{0}^{\pi} e^{z \cos t} \cos n t d t
$$

holds true.

For $n=1,2, \ldots$, the following formulas (see, e.g., [22], formulas $3.71(1)$ and (2)) hold true:

$$
\begin{aligned}
& I_{n-1}(z)-I_{n+1}(z)=\frac{2 n}{z} I_{n}(z), \\
& I_{n-1}(z)+I_{n+1}(z)=2 \frac{d I_{n}(z)}{d z} .
\end{aligned}
$$

Lemma 5.1. For $\rho>0$,

$$
\rho^{-(n+1) / 2} \int_{0}^{t} \frac{n+1}{x} e^{-(1+\rho) x} I_{n+1}(2 x \sqrt{\rho}) d x= \begin{cases}1-v_{n}(t), & \rho<1, \\ \rho^{-(n+1)}-v_{n}(t), & \rho \geqslant 1,\end{cases}
$$

where

$$
v_{n}(t)=\frac{2}{\pi \rho^{n / 2}} \int_{0}^{\pi} e^{-t A(\theta)} \frac{\sin (n+1) \theta \sin \theta}{A(\theta)} d \theta, \quad A(\theta)=1+\rho-2 \sqrt{\rho} \cos \theta .
$$

\footnotetext{
${ }^{10}$ In fact, also for complex $z$; we restrict ourselves by real positive $z$.
} 
Proof of Lemma 5.1. Applying (55), one has

$$
\begin{aligned}
\rho^{-(n+1) / 2} \int_{0}^{t} \frac{n+1}{x} e^{-(1+\rho) x} & I_{n+1}(2 x \sqrt{\rho}) d x \\
& =\rho^{-n / 2} \int_{0}^{t}\left[I_{n}(2 x \sqrt{\rho})-I_{n+2}(2 x \sqrt{\rho})\right] e^{-(1+\rho) x} d x .
\end{aligned}
$$

Applying (54) and changing the order of integration, one has further

$$
\begin{aligned}
\rho^{-n / 2} \int_{0}^{t}\left[I_{n}(2 x \sqrt{\rho})-\right. & \left.I_{n+2}(2 x \sqrt{\rho})\right] e^{-(1+\rho) x} d x \\
& =\frac{1}{\pi \rho^{n / 2}} \int_{0}^{\pi}\left(\int_{0}^{t} e^{-x A(\theta)} d x\right)(\cos n \theta-\cos (n+2) \theta) d \theta .
\end{aligned}
$$

Since $\cos n \theta-\cos (n+2) \theta=2 \sin (n+1) \theta \sin \theta$, and $\int_{0}^{t} e^{-x A(\theta)} d x=\left(1-e^{A(\theta) t}\right) / A(\theta)$,

$$
\begin{aligned}
& \frac{1}{\pi \rho^{n / 2}} \int_{0}^{\pi} \int_{0}^{t} e^{-x A(\theta)} d x[\cos n \theta-\cos (n+2) \theta] d \theta \\
& \quad=\frac{2}{\pi \rho^{n / 2}}\left(\int_{0}^{\pi} \frac{\sin (n+1) \theta \sin \theta}{A(\theta)} d \theta-\int_{0}^{\pi} e^{-t A(\theta)} \frac{\sin (n+1) \theta \sin \theta}{A(\theta)} d \theta\right) .
\end{aligned}
$$

Bearing in mind that (see, e.g., [10], equations 3.613.3)

$$
\int_{0}^{\pi} \frac{\sin n x \sin x}{1-2 a \cos x+a^{2}} d x= \begin{cases}\frac{\pi}{2} a^{n-1}, & a^{2}<1 \\ \frac{\pi}{2 a^{n+1}}, & a^{2}>1\end{cases}
$$

the proof is complete.

Note that in case of $\rho<1$ equation (57) follows directly from formulas (5.73) and (5.73a) in Section 5.7 of [17]; Riordan [17] attributed this result to Vaulot.

LEMMA 5.2. The equalities

$$
\begin{aligned}
& \int_{0}^{\infty} e^{-b x} I_{n}(a x) x^{-1} d x=\frac{a^{n}}{n\left(b+\sqrt{b^{2}-a^{2}}\right)^{n}}, \\
& \int_{0}^{\infty} e^{-b x} I_{n}(a x) d x=\frac{a^{n}}{\sqrt{b^{2}-a^{2}}\left(b+\sqrt{b^{2}-a^{2}}\right)^{n}}
\end{aligned}
$$

hold true for $b>a>0$ and $n=1,2, \ldots$

Proof of Lemma 5.2. To check (58) and (59), see, e.g., [10], equations 6.611.4, 6.623.3 and recall that $I_{n}(z)=\exp (-n \pi i / 2) J_{n}(i z)$, or [22], chapter XIII, $\S 13.2$, equations (7) and (8).

LEMMA 5.3. For $r_{n}(x)=I_{n+1}(x) / I_{n}(x)$, the inequality

$$
0 \leqslant \frac{x}{n+\frac{1}{2}+\left(x^{2}+\left(n+\frac{3}{2}\right)^{2}\right)^{1 / 2}} \leqslant r_{n}(x) \leqslant \frac{x}{n+\frac{1}{2}+\left(x^{2}+\left(n+\frac{1}{2}\right)^{2}\right)^{1 / 2}} \leqslant 1
$$

holds true for $x \geqslant 0$ and $n=0,1,2, \ldots$.

Proof of Lemma 5.3. This assertion is a particular case (for integer $n$ ) of the inequality (16) in [2]. 


\section{References}

[1] Abramowitz, M., Stegun, I.A. (1972) Handbook of Mathematical Functions, 10-th ed., Dover, New York.

[2] Amos, D.E. (1974) Computation of modified Bessel functions and their ratios. Mathematics of Computation, vol. 28, 239-251.

[3] Asmussen, S. (1984; 1985) Approximations for the probability of ruin within finite time, Scandinavian Actuarial Journal, 31-57; 64.

[4] Asmussen, S. (2000) Ruin Probabilities. World Scientific Publishers: Singapore.

[5] Barndorff-Nielsen, O., Schmidli, H. (1995) Saddlepoint approximations for the probability of ruin in finite time, Scandinavian Actuarial Journal, 169-186.

[6] Borch, K. (1967) The theory of risk, Journal of the Royal Statist. Soc., Ser. B, vol. 29, no. 3, 432-452; Discussion, ibid., 452-467.

[7] Drekic, S., Willmot, G.E. (2003) On the density and moments of the time of ruin with exponential claims, ASTIN Bulletin, vol. 33, no. 1, 11-21.

[8] Feller, W. (1971) An Introduction to Probability Theory and its Applications, vol. II, 2-nd ed., Wiley \& Sons, New York, etc.

[9] Gerber, H.U., Shiu, E.S.W. (1997) The joined distribution of the time of ruin, the surplus immediately before ruin, and the deficit at ruin, Insurance: Mathematics and Economics, vol. $21,129-137$.

[10] Gradshtein, I.S., Ryzhik, I.M. (1980) Table of Integrals, Series, and Products. Academic Press.

[11] Malinovskii, V.K. (1998) Non-poissonian claims arrivals and calculation of the probability of ruin, Insurance: Mathematics and Economics, vol. 22, 123-138.

[12] Malinovskii, V.K. (2000) Probabilities of ruin when the safety loading tends to zero, Advances in Applied Probability, vol. 32, 885-923.

[13] Malinovskii, V.K. (2003) On a non-linear dynamic solvency control model. Contribution to: XXXIV ASTIN Colloquium, Berlin, 24-27 August, 2003.

[14] Malinovskii, V.K. (2006) Risk theory insight into the asset-liability and solvency adaptive management. Contribution to 28-th International Congress of Actuaries, Paris, May, 2006.

[15] Malinovskii, V.K. (2006) Calculation of ruin probability and target capital value of an adaptive control strategy. Submitted for publication.

[16] Prabhu, N.U. (1965) Queues and Inventories. Wiley \& Sons, New York, etc.

[17] Riordan, J. (1962) Stochastic Service Systems. Wiley \& Sons, New York, etc.

[18] Seal, H.L. (1972) Numerical calculation of the probability of ruin in the Poisson/Exponential case, Mitt. Verein. Schweiz. Versich. Math., vol. 72, 77-100.

[19] Seal, H.L. (1974) The numerical calculation of $U(w, t)$, the probability of non-ruin in an interval (0,t), Scandinavian Actuarial Journal, 121-139.

[20] Takács, L. (1967) Combinatorial Methods in the Theory of Stochastic Processes, Wiley, New York, etc.

[21] Wang Rongming, Liu Haifeng (2002) On the ruin probability under a class of risk processes, ASTIN Bulletin, vol. 32, no. 1, 81-90.

[22] Watson, G.N. (1945) A Treatise on the Theory of Bessel Functions, Cambridge University Press, Cambridge.

[23] Whittaker, E.T., Watson, G.N. (1963) A Course of Modern Analysis, 4-th ed., Cambridge University Press, Cambridge.

Steklov Mathematical Institute, 119991, Gubkina Str., 8, Moscow, Russia, And FiNance Academy, 125468, Leningradsky Prosp., 49, Moscow, Russia

E-mail address: malinov@mi.ras.ru, malinov@orc.ru, malinov@fa.ru $U R L$ : http://www.mi.ras.ru/ ${ }^{m a l i n o v, ~ h t t p: / / w w w . a c t u a r i e s . f a . r u ~}$ 